\title{
The Future of Proteomics in the Study of Alcoholism
}

\author{
Chinnaswamy Kasinathan, Kent Vrana, Laura Beretta, Paul Thomas, Randy Gooch, Travis Worst, Stephen Walker, Aaron Xu,
} Peter Pierre, Heather Green, Kathleen Grant, and Paul Manowitz

\begin{abstract}
This article represents the proceedings of a workshop at the 2003 annual meeting of the Research Society on Alcoholism in Fort Lauderdale, FL. The workshop organizers/chairpersons were Chinnaswamy Kasinathan and Paul Manowitz. The presentations were (1) Introduction to the field of proteomics, by Kent Vrana; (2) Use of proteomics in the identification of urinary biomarkers for alcohol intake, by Chinnaswamy Kasinathan, Paul Thomas, and Paul Manowitz; (3) Proteomics screening illuminates ethanolmediated induction of HDL proteins in macaques, by Kent Vrana, Randy Gooch, Travis Worst, Stephen Walker, Aaron Xu, Peter Pierre, Heather Green, and Kathleen Grant; and (4) Proteomics applied to the study of the liver, by Laura Beretta.
\end{abstract}

Key Words: Proteomics, Mass Spectrometry, Biomarkers, HDL Proteins, Human Proteome Organization (HUPO).

$\mathbf{T}$ HE PURPOSE OF this workshop, The Future of Proteomics in the Study of Alcoholism, was 2-fold: (1) to present a review of proteomic methodologies that have been relatively recently developed and (2) to provide some examples of applications of these methods. The idea for this workshop was developed approximately a year ago by Chinnaswamy Kasinathan and Paul Manowitz, who were trying to find a method to identify protein changes caused by alcohol intake. As they began to discuss this research project, they realized that the tremendous recent advances in the field of proteomics offered excellent opportunities to study alcoholism. Furthermore, their cursory survey of the literature indicated that these recent advances in proteomics have not been widely applied to alcoholism research.

In great part, the field of proteomics has relied on the methodological advances of mass spectrometry. For example, before the use of mass spectrometry to sequence proteins, sequencing was performed with the Edman's reaction, which took approximately $12 \mathrm{hr}$ per sample. The same sequence determination can be obtained by mass spectrometry in approximately a second, with far greater sensitivity.

From the Oral Biology Department, University of Medicine and Dentistry of New Jersey, Newark, New Jersey (CK); Department of Physiology and Pharmacology, Wake Forest University School of Medicine, Winston-Salem, North Carolina (KV, RG, TW, SW, AX, PP, HG, KG); Department of Microbiology and Immunology, University of Michigan, Ann Arbor, Michigan (LB); Department of Chemical Biology, Laboratory for Cancer Research, Rutgers, Piscataway, New Jersey (PT); and Department of Psychiatry, University of Medicine and Dentistry of New Jersey-Robert Wood Johnson Medical School, Piscataway, New Jersey (PM).

Received for publication September 22, 2003; accepted November 4, 2003.

Reprint requests: Chinnaswamy Kasinathan, PhD, Oral Biology Department, University of Medicine and Dentistry of New Jersey, 110 Bergen St., Newark, NJ 07103; Fax: 973-972-0045; E-mail: kasinach@umdnj.edu.

Copyright () 2004 by the Research Society on Alcoholism.

DOI: 10.1097/01.ALC.0000113779.35260.A8
Once sequences have been obtained, these sequences can be compared with the databases of known sequences to identify the proteins. In this regard, the sequencing of the human genome has greatly facilitated the progress made in proteomics.

Because of the very rapid growth in this area, there are many proteomic facilities throughout the world. Therefore, this workshop was particularly valuable for researchers who do not have a mass spectrometer but might want to collaborate with a proteomic center to solve some of their scientific problems in alcoholism. The topics that were addressed in this workshop included (1) identification of biomarkers of alcohol intake, (2) delineation of biochemical pathways to understand alcohol's effects, and (3) a study of hepatic proteins, including a discussion of the Human Proteome Organization.

\section{INTRODUCTION TO THE FIELD OF PROTEOMICS}

\section{Kent Vrana}

The completion of the human genome project and recent advances in technology have provided the alcohol research community with a wide array of new technologies. The flow of genetic information provides us with (1) genomics-the study of the sequence of the genetic material (the genome); (2) transcriptomics - the characterization of the complement of messenger RNA molecules expressed in a tissue or cell; (3) proteomics-examination of the proteins that are synthesized by a cell, tissue, or organ; and even (4) metabolomics - the collection of small molecule metabolites that are indicative of health and disease (lipid mediators, signal transduction molecules, and so on). This discussion is focused on the penultimate of these-proteomics.

The central premise of proteomics is that the complete characterization of the proteins in a cell, tissue, or organ 
will provide insights into the status of the system. That is to say, a detailed understanding of the levels, structures, activities, and interactions of the proteins will serve as a molecular correlate of the physiologic state of the system. This is a particularly cogent point when one realizes that the DNA and messenger RNA in a system do not, in fact, directly represent the working aspects of the cell. For instance, although a mutation, or polymorphism, within a cell might be associated with a disorder, it is the altered level or function of the encoded protein that actually determines the result. So why is it that we bother to pursue genomic (gene-sequencing) or transcriptomic (DNA microarrays) studies? The answer is that we have the technologies to perform the former studies, whereas characterization of the complete proteome remains a technological hurdle. This workshop provides an overview of aspects of this technological approach.

Proteomics can be divided into three major components based on methodological considerations. Structural proteomics is the study of the physical arrangement of amino acids into second-, third-, and fourth-order relationships. In essence, because structure determines function, this is the most elemental level of proteomics. This field would typically use technologies such as x-ray crystallography and NMR spectroscopy. Functional proteomics addresses the actual physiologic activity of proteins, whether it be enzyme activity, physiologic interactions between proteins, or interactions with other environmental constituents (e.g., lipids or nucleic acids). Enzyme activity assays, receptor binding, and protein-protein interactions are examples of these experimental approaches. Finally, expression proteomics represents the emerging field of study that focuses on the patterns of protein expression levels in health and disease. It is this form of proteomics that is receiving the most attention in recent years and that is the subject of the papers presented in this symposium. Notably, this field of study has been enabled by the advent of new highthroughput technologies for separating proteins in time and space (by using two-dimensional gel electrophoresis, differential protein capture, or sophisticated liquid chromatography technologies) and their subsequent quantification and identification (typically via mass spectrometry). Although the presentations in this symposium focus on medium- to high-throughput screening of expression proteomics, we do not intend to suggest that multiplex analysis of structural and functional proteomics is not possible or that it is not equally important. In fact, the highthroughput, robotic analysis of small molecule inhibitors and activators of enzymes is the basis for much of the pharmaceutical industry's drug discovery program. In addition, efforts to catalog all of the structural motifs present in the field of structural proteomics promise to dramatically increase the speed and fidelity of computer protein modeling and characterization.

Together, these rapidly evolving technologies for the characterization of proteins promise to provide a wealth of information on health and disease. Indeed, as was shown in the subsequent presentations in this symposium, we are already gaining new insights into the pathophysiology of alcohol.

\section{USE OF PROTEOMICS IN THE IDENTIFICATION OF URINARY BIOMARKERS FOR ALCOHOL INTAKE}

\section{Chinnaswamy Kasinathan, Paul Thomas, and Paul Manowitz}

Alcoholism is a treatable disease (McCrady and Langenbucher, 1996; Weinrieb and O'Brien, 1997). Furthermore, early detection of high alcohol intake can result in a reduction of alcohol intake and psychosocial problems and in prevention of alcoholism. It is well known that alcoholics often deny their drinking habit and are able to hide their level of consumption. Therefore, a convenient method to detect excessive ethanol intake, by itself or in conjunction with other detection methods, would be helpful for monitoring alcoholics in treatment and for identifying subjects who are at risk for alcoholism.

Biomarkers are being increasingly used to evaluate the effectiveness of alcoholism treatment (Anton et al., 1999; Chick et al., 1992; Johnson et al., 1996; Volpicelli et al., 1997). However, research during the past 15 years has not provided a perfectly valid biomarker. The most commonly used biomarker tests-the carbohydrate-deficient transferrin and $\gamma$-glutamyl transpeptidase tests-are useful for identifying individuals with alcohol problems, but they have significant limitations (Lakshman et al., 2001; Litten et al., 1995). Furthermore, these biomarkers do not identify alcohol problems for women as well as they do for men. In addition, these tests perform well on adults but not on adolescents with alcohol problems. It is clear that a more valid biomarker is needed to prevent and treat alcohol abuse and alcoholism in all groups. Also, the available biomarkers are generally blood based and, therefore, have the limitation of requiring phlebotomy.

To identify a urinary biomarker for alcohol intake, male Sprague-Dawley rats were subjected to pair feeding with regular Lieber-DeCarli liquid diets and a low-fat ethanol diet (Dyets, Inc., Bethlehem, PA). Each control animal was paired with an ethanol-treated animal so that the number of calories consumed by the ethanol-treated rat was the amount the control received on the following day. The animals were initially fed with control diet for 2 days. Pair feeding was started with $3 \%$ ethanol on day $3,4 \%$ ethanol on day 4 , and $5 \%$ ethanol for the remaining 15 days of treatment.

Analysis of the urine samples from ethanol-treated and control rats was performed by using two-dimensional gel electrophoresis with Coomassie blue staining. The twodimensional electrophoretic separation revealed the presence of several unique proteins that were present in the urine of the ethanol-treated animals but were absent in the urine of control animals. In one region of the two- 
dimensional gel of the urine from ethanol-treated animals, there were two groups of proteins: one group contained three proteins with a molecular weight of approximately 70 $\mathrm{kDa}$ separated by different isoelectric points, and another group contained three proteins with a molecular weight of approximately $66 \mathrm{kDa}$ separated by different isoelectric points. These 6 bands were very prominent and distinct and have been observed in urine samples from 14 ethanoltreated rats. The protein-stained spots were cut from the gel as 3-mm cylindrical plugs and destained with several changes of acetonitrile:water (6:4). These plugs were taken to near dryness and rehydrated in ammonium carbonate buffer containing modified trypsin. After overnight incubation at $37^{\circ} \mathrm{C}$, the peptides resulting from trypsin hydrolysis were recovered from the buffer and concentrated by sequential absorption and elution with ZipTips (Millipore Corp., Bedford, MA). ZipTips are small quantities of reverse-phase $\mathrm{C}-18$ resin packed in pipet tips. Mass spectra analysis of these peptides reveals the molecular weights of the individual peptides, and these are allowed to fragment in an additional mass-resolving part of the same mass spectrometry (MS) instrument (MS-MS, i.e., tandem MS) to yield fragmentation patterns of the peptides. The fragmentation patterns obtained by these MS-MS instruments (LCQ DECA XP Ion Trap, Thermo Electron Corporation, West Palm Beach, FL) are a rich source of information that is dependent on the sequence of amino acids in the particular peptide. These complex fragmentation patterns are then compared with databases of amino acid sequence information that are derived from protein and DNA sequence databases. When there is a relatively good agreement of the MS-MS data with the expected fragmentation pattern of the peptide in the database, then it is reasonable to assume that it is the same protein or a closely related family member. It should be appreciated that this type of so-called identification of the protein or proteins in a twodimensional gel spot is dependent both on the completeness of the data in the database (including the absence of errors) and on the quality of the MS-MS data. Clearly, if an important concept hinges on the identification of a protein spot, there should be some secondary support. For example, recognition of the blotted spot by a specific antibody or some property of its regulation of expression or unique subcellular distribution would serve to build a consensus of agreement as to its identity.

One of the bands, identified as transferrin by mass spectrometry, was present in urine from the ethanol-fed rat, but not in that of the control rat. Additional studies are ongoing to identify the other bands specific to the urine from alcohol-treated animals.

The ultimate goal of this research is to apply what is known from rats to the development of good urinary biomarkers in humans as a measure of alcohol intake. This would have particular application in many alcoholism treatment facilities. The National Survey of Substance Abuse Treatment Services (National Directory of Drug and Alco- hol Abuse Treatment Programs, 2000) listed approximately 17,000 facilities that treated patients with substance abuse, including alcoholism. The nature of these facilities varied widely from very small solo practices to very large hospital complexes and residential treatment centers. Many of these facilities do not have easy access to phlebotomists on site. Therefore, urinary tests are more convenient measures of alcohol intake than blood tests in many of these facilities.

\section{PROTEOMICS SCREENING ILLUMINATES ETHANOL- \\ MEDIATED INDUCTION OF HDL [HIGH-DENSITY LIPOPROTEIN] PROTEINS IN MACAQUES}

Kent Vrana, Randy Gooch, Travis Worst, Stephen Walker, Aaron Xu, Peter Pierre, Heather Green, and Kathleen Grant

Alcohol abuse and alcoholism remain serious societal problems with wide-ranging effects on biochemistry, physiology, and behavior. In this study, cynomolgus monkeys (Macaca fascicularis) were used in an ethanol selfadministration protocol for proteomic serum profiling. Surface-enhanced laser desorption ionization time-of-flight (SELDI-TOF) mass spectrometry and other proteomic methodologies were used to identify serum biomarkers of the consumption of ethanol. Serum samples from monkeys trained to self-administer ethanol $(4 \% \mathrm{w} / \mathrm{v}$ prepared with water only) were collected from the ethanol-naive state through 9 months of heavy ethanol-consumption and then into a period of enforced abstinence. Samples were subjected to analysis on SELDI-TOF hydrophobic ProteinChips (Ciphergen Biosystems, Inc., Fremont, CA) to illuminate differentially expressed proteins. Potential biomarkers were then identified by using one- and twodimensional polyacrylamide gel electrophoresis and subsequent trypsin digestion and mass spectroscopic fingerprint identification of the excised bands of interest. On analysis of SELDI-TOF mass spectra, two prominent serum proteins, one at $27.9 \mathrm{kDa}$ and another at $8.7 \mathrm{kDa}$, were observed to be differentially expressed. Analysis of the 27.9and $8.7-\mathrm{kDa}$ proteins by trypsin digestion fingerprinting identified them as apolipoprotein AI and apolipoprotein AII, respectively. Their correspondence with the SELDITOF MS peak was verified with purified human apolipoprotein AI and AII proteins. Immunoblot analysis of samples from individual animals confirmed that ethanol consumption significantly increased apolipoprotein AII. However, although apolipoprotein AI seemed to increase after ethanol self-administration, wide interanimal variability precluded a statistically significant finding. In agreement with previous studies of human ethanol consumption, these studies suggest that alcohol self-administration produces an increase of lipoproteins associated with cardiovascular protective effects. These findings contribute further evidence that it is the ethanol itself that elicits these potential beneficial effects. Moreover, this medium-throughput analysis of several hundred proteins (with the identification of two 
important species) points to the promise of proteomic screening as a tool to better understand the pathophysiological characterization of alcohol abuse and alcoholism.

\section{PROTEOMICS APPLIED TO THE STUDY OF THE LIVER}

\section{Laura Beretta}

With the completion of the genome project, the proteome has been viewed as the next major challenge. There is a need to begin an organized effort whose goals include developing an infrastructure in proteomics that would substantially facilitate unraveling the complexity of the proteome in health and disease. The Human Proteome Organization (http://www.hupo.org) was founded to regroup scientists in the public and private sectors engaged throughout the world in various aspects of proteomics. The Human Proteome Organization has outlined a mission for itself to (1) consolidate national and regional proteome organizations into a worldwide organization; (2) engage in scientific and educational activities to encourage the spread of proteomics technologies and disseminate knowledge pertaining to the human proteome and that of model organisms; and (3) assist in the coordination of public proteome initiatives aimed at comprehensively characterizing specific tissue and cell proteomes. Initiatives currently in the piloting phase include an international effort to identify proteins detectable in normal serum and plasma and their range of variation with age, ethnicity, and physiologic states and a liver proteome initiative to identify proteins expressed in the liver. These initiatives have attracted substantial interest and will be integrated with efforts in protein informatics to achieve data standardization on the one hand and data curation on the other. The strongest and most compelling argument for a liver proteome initiative is the essential and multifunction role of the liver in human health and disease. Liver disease of some type affects more than $10 \%$ of the world population. Liver pathogenesis remains, however, largely undefined, and most liver diseases remain poorly diagnosed, staged, and treated. In addition, many proteins in plasma are synthesized in the liver and change with disease in the liver, as well as with systemic responses to inflammation and other disease processes in other organs. Finally, protein signatures in preclinical study models and in human liver samples could be used as an effective way to understand pharmacology and toxicology. Understanding the liver proteome will accelerate the development of diagnostics and therapeutics toward the diseases of this organ and also facilitate drug discovery. The importance of liver proteomics research is reflected by the commitment of countries worldwide to support in a major way such an initiative. Scientific objectives of the human liver proteome project include (1) a comprehensive analysis of human liver protein constituents in health and disease, with an initial focus on liver fibrosis and cancer; (2) bridging the liver proteome project and the plasma proteome project to discover biomarkers; and (3) constructing a knowledge database (integration and correlation of the human liver proteome with the liver transcriptome and the human genome). Other topics will include the determination of protein localization and posttranslational modifications of the liver proteome, the subcellular proteome, and networks of liver proteome. The immediate goal for human liver proteome project (HLPP) is to demonstrate what can be done in a pilot project. The key to the pilot study is to find one that lays a foundation for future studies. The proteome will be studied for the next 10 to 20 years or more. The hope is to stimulate that research now.

My group has recently applied proteomic technologies for biomarker discovery. Hepatocellular carcinoma (HCC) has a poor prognosis, with 5-year survival rates of less than $5 \%$. Most cases of HCC are associated with cirrhosis, which is commonly caused by alcohol intake, viral hepatitis, or both. We combined two-dimensional polyacrylamide gel electrophoresis and DNA microarray analysis to identify genes and proteins that are potentially important in the development and progression of hepatic carcinoma and that are potential new markers for early cancer diagnosis. Testing and validating these potential markers require high-affinity probes for their detection and quantitation. For this purpose, we are developing an antibody-based microarray for the diagnosis of liver cancer through serum assays. We also have recently implemented a proteomicbased approach for the identification of tumor antigens that induce an antibody response; we have applied this approach to HCC. In contrast to approaches for identification of tumor antigens on the basis of analysis of recombinant proteins, the proteomic approach we have used allows identification of autoantibodies to proteins in lysates prepared from tumors and tumor cell lines and thus may more readily uncover antigenicity associated with posttranslational modification. We uncovered a distinct repertoire of autoantibodies that characterize the humoral response in HCC. The detection of autoantibodies directed against HCC-associated antigens we have identified may have value for HCC screening, diagnosis, or follow-up.

\section{SUMMARY}

The study of proteins involved in alcoholism has involved two different approaches. One approach, the "candidate protein" approach, is to select a protein for study because of its role in a pathway postulated to involve alcoholism. Because alcoholism is a behavioral disorder, many of the candidate proteins selected for study are involved in neurochemical pathways. This is a legitimate approach that has yielded significant results (e.g., Kasinathan et al., 1998). An alternative proteomic, approach is to study as many proteins as can be separated by a technique such as twodimensional gel electrophoresis and identified through mass spectrometry. This approach does not select any candidate protein in advance as being important. Rather, this approach incorporates the entire proteosome of the body.

In this workshop, Dr. Vrana presented some of the newer proteomic methods that have been developed to separate and identify proteins. Dr. Kasinathan and associates used a pro- 
teomic approach to identify urinary proteins that are excreted by ethanol-treated rats, but not by control rats. One of the proteins, transferrin, was observed in the urine of ethanoltreated rats and not in that of the controls. Dr. Vrana and colleagues demonstrated that high-density lipoproteins in serum were increased in ethanol-treated macaques, which suggests that this may be a mechanism for a beneficial consequence of moderate ethanol intake. Dr. Beretta described the Human Proteome Organization and its efforts to characterize the liver proteome. This project may have important implications for researchers studying ethanol-induced liver disease.

The presentations given at the workshop give a glimmer of what the future may hold. The new proteomic techniques offer immense opportunities to study a large number of proteins in a single preparation that may be influenced by alcoholism. There is a saying that when the dust settles, we will find out if we are riding a horse or an ass. It would be of interest to have another meeting on proteomics and alcoholism in a decade to see what has or has not been revealed.

\section{REFERENCES}

Anton RF, Moak DH, Waid LR, Latham PK, Malcolm RJ, Dias JK (1999) Naltrexone and cognitive behavioral therapy for the treatment of out- patient alcoholics: results of a placebo-controlled trial. Am J Psychiatry 156:1758-1764.

Chick J, Gough K, Falkowski W, Kershaw P, Hore B, Mehta B, Ritson B, Ropner R, Torley D (1992) Disulfiram treatment of alcoholism. Br J Psychiatry 161:84-89.

Johnson BA, Jasinski DR, Galloway GP, Kranzler H, Weinreib R (1996) Ritanserin in the treatment of alcohol dependence-a multi-center clinical trial. Ritanserin Study Group. Psychopharmacology 128:206215.

Kasinathan C, Ramaprasad P, William S, Espina N (1998) Stimulation of tyrosylprotein sulfotransferase activity by ethanol: role of increased enzyme level. Alcohol 15:271-276.

Lakshman R, Tsutsumi M, Ghosh P, Takase S, Anni H, Nikolaeva O, et al. (2001) Alcohol biomarkers: clinical significance and biochemical basis. Alcohol Clin Exp Res 25:67S-70S.

Litten RZ, Allen JP, Fertig JB (1995) Gamma-glutamyl trans-peptidase and carbohydrate deficient transferrin: alternative measures of excessive alcohol consumption. Alcohol Clin Exp Res 19:1541-1546.

McCrady BS, Langenbucher JW (1996) Alcohol treatment and health care system reform. Arch Gen Psychiatry 53:737-746.

National Directory of Drug and Alcohol Abuse Treatment Programs (2000) Substance Abuse and Mental Health Service Administration, Rockville, MD.

Volpicelli JR, Rhines KC, Rhines JS, Volpicelli LA, Alterman AI, O’Brien CP (1997) Naltrexone and alcohol dependence. Arch Gen Psychiatry 54:737-742.

Weinrieb RM, O'Brien CP (1997) Naltrexone in the treatment of alcoholism. Annu Rev Med 48:477-487. 